\title{
UNIVERSAL LAURENT SERIES ON DOMAINS OF INFINITE CONNECTIVITY
}

\author{
VASSILI NESTORIDIS \\ (Communicated by Mario Bonk)
}

\begin{abstract}
We establish the existence of universal Laurent series on some domains of infinite connectivity. This phenomenon is topologically and algebraically generic.
\end{abstract}

\section{INTRODUCTION}

If $\Omega \subset \mathbb{C}$ is a domain and $\zeta \in \Omega$, then a holomorphic function $f \in H(\Omega)$ belongs to the class $U(\Omega, \zeta)$ of universal Taylor series with center $\zeta$ if the sequence $S_{n}(f, \zeta)(z)=\sum_{j=0}^{n} \frac{f^{(j)}(\zeta)}{j !}(z-\zeta)^{j}, n=0,1,2, \ldots$, has the following property:

For every compact set $K \subset \mathbb{C}, K \cap \Omega=\emptyset$, with connected complement and every function $h: K \rightarrow \mathbb{C}$ which is continuous on $K$ and holomorphic in $K^{0}$, there exists a sequence $\lambda_{n} \in\{0,1,2, \ldots\}, n=1,2, \ldots$, so that $S_{\lambda_{n}}(f, \zeta) \rightarrow h$ uniformly on $K$, as $n \rightarrow+\infty$.

If $\Omega$ is any simply connected domain, then the class $U(\Omega, \zeta)$ is non-empty, in fact residual in $H(\Omega)$ endowed with the topology of uniform convergence on compact subsets of $\Omega([14,9])$. We can even in addition obtain $S_{\lambda_{n}}(f, \zeta) \rightarrow f$ uniformly on compact subsets of $\Omega$.

In some unbounded non-simply connected domains $\Omega$ it may happen that $U(\Omega, \zeta)$ is non-empty ([2], 16], 10], 15]); however, in an annulus $U(\Omega, \zeta)=\emptyset([4)$. In the case $\Omega=\mathcal{D}(0,1,2)=\{z \in \mathbb{C}: 1<|z|<2\}$ it is natural to seek universal approximations on compact sets $K \subset \mathbb{C}, K \cap \mathcal{D}(0,1,2)=\emptyset$, with connected complement and not containing 0 by Laurent partial sums of a holomorphic function $f \in H(\mathcal{D}(0,1,2)), f(z)=\sum_{-\infty}^{+\infty} a_{n}(f) z^{n}$. Then the operators realizing universal approximation are of the form $T_{n}(f)=\sum_{j=-n}^{n} a_{j}(f) z^{j}, n=0,1,2, \ldots$. The existence of such universal Laurent series on an annulus was established in [3], and the corresponding class of universal Laurent series was proved to be residual in $H(\mathcal{D}(0,1,2))$. More generally, if $\Omega \subset \mathbb{C}$ is a domain of finite connectivity, it is possible to have Laurent expansions of any $f \in H(\Omega)$ and the class of universal Laurent series is residual ([3]; see also [8]). More precisely, suppose that the components of $(\mathbb{C} \cup\{\infty\}) \backslash \Omega$ are $F_{0}, F_{1}, \ldots n$ with $\infty \in F_{0}$. Then because the $F_{\ell}$ 's are at a positive distance from each other, we can find closed curves $\gamma_{0}, \gamma_{1}, \ldots, \gamma_{n}$ in $\Omega$ where $\gamma_{\ell}$ is close to the boundary of $F_{\ell}, \ell=0, \ldots, n$. We can arrange it so that $\gamma_{0}+\gamma_{1}+\cdots+\gamma_{n}$ is $\Omega$-homologous to zero and that $\operatorname{Ind}\left(\gamma_{0}+\gamma_{1}+\cdots+\gamma_{n}, z\right)=1$ for all $z$ in a compact set $L \subset \Omega$. Then by the Cauchy formula $f(z)=\sum_{j=0}^{n} \frac{1}{2 \pi i} \int_{\gamma_{j}} \frac{f(\zeta)}{\zeta-z} d \zeta$.

Received by the editors December 15, 2011 and, in revised form, September 30, 2012. 2010 Mathematics Subject Classification. Primary 30K05. 
For $j=1, \ldots, n$ the function $f_{j}(z)=\frac{1}{2 \pi i} \int_{\gamma_{j}} \frac{f(\zeta)}{\zeta-z} d \zeta$ is easily seen to be holomorphic in $(\mathbb{C} \cup\{\infty\}) \backslash F_{j}$ and satisfies $f_{j}(\infty)=0$. If we fix $c_{j} \in F_{j}, j=1, \ldots, n$, then in a neighborhood of $\infty$ we have a Laurent expansion $f_{j}(z)=\sum_{m=1}^{\infty} \frac{a_{m}\left(f_{j}\right)}{\left(z-c_{j}\right)^{m}}$, $j=1, \ldots, n$. Concerning $f_{0}(z)=\frac{1}{2 \pi i} \int_{\gamma_{0}} \frac{f(\zeta)}{\zeta-z} d \zeta$, this is holomorphic in the simply connected domain $F_{0}^{c}$ and we may consider Taylor expansions of it with respect to some center $\zeta \in F_{0}^{c}$. We consider the denumerable family $\frac{a_{m}\left(f_{j}\right)}{\left(z-c_{j}\right)^{m}}, j=1, \ldots, n$, $m=1,2, \ldots$, and $\frac{f_{0}^{(\ell)}(\zeta)}{\ell !}(z-\zeta)^{\ell}, \ell=0,1,2, \ldots$; we order it in an arbitrary way, say $e_{s}, s=0,1, \ldots$.

Then the operators realizing approximations on compact sets $K \subset \mathbb{C}, K \cap \Omega=\emptyset$, $K^{c}$ connected, $c_{j} \notin K$ for every $j=1, \ldots, n$ are the partial sums $T_{N}(f)=\sum_{s=0}^{N} e_{s}$.

It is important to say that the components $f_{0}, f_{1}, \ldots, f_{n}$ of $f$ are uniquely determined, because if we have another set $\widetilde{f}_{0}, \widetilde{f}_{1}, \ldots, \tilde{f}_{n}$ of such functions, then $f_{0}+f_{1}+\cdots+f_{n}=f=\tilde{f}_{0}+\tilde{f}_{1}+\cdots+\tilde{f}_{n}$ on $\Omega$, which implies $f_{0}-\widetilde{f}_{0}=$ $\left(\widetilde{f}_{1}-f_{1}\right)+\cdots+\left(\widetilde{f}_{n}-f_{n}\right)$. The left member is holomorphic in $F_{0}^{c}$. The right member is holomorphic in a neighborhood of $F_{0}$ and vanishes at $\infty$. Thus, we have an entire function with a zero at $\infty$. By Liouville's Theorem, $f_{0}-\widetilde{f}_{0} \equiv 0$, and continuing in this way $f_{1}-\widetilde{f}_{1} \equiv 0, \ldots, \widetilde{f}_{n}-f_{n} \equiv 0$. Therefore, the components $f_{0}, f_{1}, \ldots, f_{n}$ are uniquely determined by $f$. They also depend continuously on $f$, because they are given by integrals of $f$ on compact sets. This implies that the set of universal Laurent series (in the finitely connected domain $\Omega$ ) is a $G_{\delta}$. Runge's Theorem combined with Baire's Category Theorem ([7], [6]) imply density.

In the present paper we will consider the case of some infinitely connected domains $\Omega$ and we will establish the existence of universal Laurent series. If $F=(\mathbb{C} \cup\{\infty\}) \backslash \Omega$ has a component $F_{1} \subset \mathbb{C}$ which is compact and far from $F \backslash F_{1}$, then it can be surrounded by a closed curve $\gamma_{1}$ in $\Omega$ and we can define the Cauchy transform $f_{1}(z)=\frac{1}{2 \pi i} \int_{\gamma_{1}} \frac{f(\zeta)}{\zeta-z} d \zeta$, which is holomorphic in $(\mathbb{C} \cup\{\infty\}) \backslash F_{1}$ and vanishes at $\infty$. In a similar way, if we have other such components $F_{2}, F_{3}, \ldots$ we define the components $f_{2}, f_{3}, \ldots$ of $f$. They are uniquely determined by $f$, because for every $n \in \mathbb{N}=\{1,2,3, \ldots\}$ we have $f=f_{1}+f_{2}+\cdots+f_{n}+R_{n}$, where $f_{\ell}$ is holomorphic in $(\mathbb{C} \cup\{\infty\}) \backslash F_{\ell}$ and vanishes at $\infty$, and where $R_{n}$ is holomorphic in a neighborhood of $F_{1} \cup \cdots \cup F_{n}$ and in $\Omega$. An argument using Liouville's Theorem gives that the components $f_{1}, \ldots, f_{n}$ are uniquely determined by $f$ and this holds for every $n \in \mathbb{N}$. Because $f_{n}$ is given as an integral of $f$ on a compact set, $f_{n}$ depends continuously on $f$. Further, we fix $c_{j} \in F_{j}, j=1,2, \ldots$, and we develop $f_{j}$ in a Laurent series with center $c_{j}$ in a neighborhood of $\infty$. It is well-known that the Laurent coefficients of $f_{j}$ vary continuously with respect to $f_{j}$ (then by composition they vary continuously with respect to $f$ ).

There are denumerably many terms appearing in this way, and we can order them to take the form $e_{s}=e_{s}(f), s=0,1,2, \ldots$. Then the operators realizing approximations will be the partial sums $T_{N}(f)=\sum_{s=0}^{N} e_{s}(f)$ or subsequences of them.

The approximations will be on compact sets $K \subset \mathbb{C}, K \cap \Omega=\emptyset$, with connected complement which will be at a strictly positive distance from all poles $c_{j}, j=$ $1,2, \ldots$.

The continuous dependence on $f$ will guarantee that the set of universal series will be a $G_{\delta}$. Density will follow by a combination of Runge's Theorem and Baire's 
Theorem. The question is whether we can call these universal series Laurent series. A weak point is that the difference $R_{n}=f-\left(f_{1}+\cdots+f_{n}\right)$ may not tend to 0 .

In fact, using the Mittag-Leffler Theorem we can give examples where the sequence $R_{n}\left(z_{0}\right), n=1,2, \ldots$, has more than one accumulation point. Indeed, there exists a function $f$ holomorphic in $\mathbb{C}-\{1,2, \ldots\}$ such that for every $n \in\{1,2, \ldots\}$, the principal part is $P_{n}(z)=\frac{n \cdot(-1)^{n}}{z-n}$. Then $f_{n}=P_{n}$ and $R_{n}=f-\left(f_{1}+\cdots+f_{N}\right)$ has two accumulation points at $z_{0}=0$. Thus, $R_{n}$ does not converge to zero. So we cannot speak about a Laurent expansion. However, we can strengthen our results in order to simultaneously obtain the approximation $T_{\lambda_{n}}(f) \rightarrow f$ in $\Omega$. This perhaps justifies the term universal Laurent series.

The topological requirements on $\Omega$ so that the previous procedure works are the following:

Condition (A): $\quad \Omega=(\mathbb{C} \cup\{\infty\}) \backslash F \quad$ is a domain. Among the components of $F$ we can find a sequence $F_{1}, F_{2}, \ldots$ so that $F_{j} \subset \mathbb{C}$ is compact for $j=1,2, \ldots$ and $\overline{\bigcup_{j=1}^{\infty} F_{j}}=F$, where the closure is taken in $\mathbb{C} \cup\{\infty\}$ and each $F_{\ell}, \ell=1,2, \ldots$, is far from $F-F_{\ell}$; that is, there exists $\delta_{\ell}>0$ so that $\operatorname{dist}\left(F_{\ell}, F_{j}\right)>\delta_{\ell}$ for all $j \neq \ell$, where the distance is computed in chordal metric in $\mathbb{C} \cup\{\infty\}$.

The simplest case is when the $F_{\ell}$ are discs or segments or singletons accumulating only at $\infty$ (then $\infty \in F$ ). However, we can also have finite accumulation points and we can have $\infty \notin F$ (in which case $\infty \in \Omega$ ).

Certainly the case where $F$ is a Cantor set is not covered by our considerations.

Further, in each $F_{\ell}$ we select a pole $c_{\ell} \in F\left(c_{\ell} \neq \infty\right)$. The universal approximations will be realized on compact sets $K \subset \mathbb{C}, K \cap \Omega=\emptyset$ with connected complement, with $K \cap \overline{\left\{c_{\ell}, \ell=1,2, \ldots\right\}}=\emptyset$.

This is the first example of domains of infinite connectivity where universal Laurent series exist.

\section{Preliminary Results}

We need the following topological lemma from the master's thesis of N. Tsirivas (in Greek), which extends a well-known lemma; see also [5] and [2].

Lemma 2.1. Let $K \subset \mathbb{C} \cup\{\infty\}$ be compact and $A \subset \mathbb{C} \cup\{\infty\}$ be a set intersecting every component of $(\mathbb{C} \cup\{\infty\}) \backslash K$. Let $V \subset \mathbb{C} \cup\{\infty\}$ be open such that $K \subset$ $V$. Then there exists $W$ open such that $K \subset W \subset V$ and every component of $(\mathbb{C} \cup\{\infty\}) \backslash W$ intersects $A$.

Proof. Let $\rho$ denote the chordal metric in $\mathbb{C} \cup\{\infty\}$. The disjoint compact sets $K$ and $(\mathbb{C} \cup\{\infty\}) \backslash V$ have a strictly positive distance $\operatorname{dist}_{\rho}(K,(\mathbb{C} \cup\{\infty\}) \backslash V)=$ $\xi>0$. Then the family of $\operatorname{discs} D_{\rho}\left(z, \frac{\xi}{2}\right)=\left\{w \in \mathbb{C} \cup\{\infty\}: \rho(w, z)<\frac{\xi}{2}\right\}$ where $z \in(\mathbb{C} \cup\{\infty\}) \backslash V$ is an open cover of the compact set $(\mathbb{C} \cup\{\infty\}) \backslash V$. Therefore there are $z_{1}, \ldots, z_{N} \in(\mathbb{C} \cup\{\infty\}) \backslash V$ such that

$$
(\mathbb{C} \cup\{\infty\}) \backslash V \subset \bigcup_{\ell=1}^{N} D_{\rho}\left(z_{\ell}, \frac{\xi}{2}\right) \subset \bigcup_{\ell=1}^{N} \overline{D_{\rho}\left(z_{\ell}, \frac{\xi}{2}\right)} \subset(\mathbb{C} \cup\{\infty\}) \backslash K .
$$

The component of $z_{\ell}$ inside $(\mathbb{C} \cup\{\infty\}) \backslash K$ contains at least one point $w_{\ell} \in A$, by assumption. This component is a domain in $\mathbb{C} \cup\{\infty\}$ and contains the points $z_{\ell}, w_{\ell}$. Therefore there exists a polygonal line $\Gamma_{\ell}$ inside this component starting at 
$z_{\ell}$ and ending at $w_{\ell}$ (if $\infty$ is a vertice of $\Gamma_{\ell}$, then the sides of $\Gamma_{\ell}$ ending at $\infty$ will be straight half lines with $\infty$ ).

The set $B=\bigcup_{\ell=1}^{N}\left[\overline{D_{\rho}\left(z_{\ell}, \frac{\xi}{2}\right)} \cup \Gamma_{\ell}\right]$ is closed and satisfies $(\mathbb{C} \cup\{\infty\}) \backslash V \subset$ $B \subset(\mathbb{C} \cup\{\infty\}) \backslash K$. We set $W=(\mathbb{C} \cup\{\infty\}) \backslash B$, which is an open set and satisfies $(\mathbb{C} \cup\{\infty\}) \backslash W=B=\bigcup_{\ell=1}^{n}\left[\overline{D_{\rho}\left(z_{\ell}, \frac{\xi}{2}\right)} \cup \Gamma_{\ell}\right]$ and $K \subset W \subset V$. Since $z_{\ell} \in \Gamma_{\ell} \cap \overline{D_{\rho}\left(z_{\ell}, \frac{\xi}{2}\right)}$, the set $\overline{D_{\rho}\left(z_{\ell}, \frac{\xi}{2}\right)} \cup \Gamma_{\ell}$ is connected. Therefore, every component of $(\mathbb{C} \cup\{\infty\}) \backslash W$ contains a $\Gamma_{\ell}$ which meets $A$, since $w_{\ell} \in \Gamma_{\ell} \cap A$.

The proof is complete.

We consider a compact set $F \subset \mathbb{C} \cup\{\infty\}$, which may contain $\infty$ or not. We assume that the open set $\Omega=(\mathbb{C} \cup\{\infty\}) \backslash F$ is connected and therefore is a domain in $\mathbb{C} \cup\{\infty\}$. We also assume that among the components of $F$ we may choose denumerably many $F_{\ell}, \ell=1,2, \ldots$, so that Condition (A) is satisfied.

Finally, we fix a point $c_{\ell} \in F_{\ell}, \ell=1,2, \ldots$, and we set $\Gamma=\overline{\left\{c_{\ell} ; \ell=1,2, \ldots\right\}}$, where the closure is taken in $\mathbb{C} \cup\{\infty\}$. We observe that $c_{\ell} \neq \infty$ for all $\ell=1,2, \ldots$ and $\Gamma \subset F$. If $\infty \notin F$, then $\infty \notin \Gamma$ and $\infty \in \Omega$. If $\infty \in F$, then $\infty \notin \Omega$ and $\infty$ may belong to $\Gamma$ or not.

Under the above assumptions we have the following lemma of a topological nature which may be compared with other topological lemmas in [13, [9], 3].

Lemma 2.2. There exists a sequence of compact sets $K_{m} \subset \mathbb{C}, m=1,2, \ldots$, with $K_{m} \cap(\Omega \cup \Gamma)=\emptyset$ and $K_{m}^{c}$ connected such that every compact set $K \subset \mathbb{C}$ with $K \cap(\Omega \cup \Gamma)=\emptyset$ and $K^{c}$ connected is included in some $K_{m}$.

Proof. We fix $\zeta_{0} \in \Omega \cap(\mathbb{Q}+i \mathbb{Q})$. Let $K \subset \mathbb{C}$ be compact with $K \cap(\Omega \cup \Gamma)=\emptyset$ and $K^{c}$ connected. Then there exists $n \in \mathbb{N}$ so that $|z| \leq n$ for all $z \in K$. There also exists $s \in\{1,2, \ldots\}$ so that $\operatorname{dist}_{\rho}(K, \Gamma)>\frac{1}{s}$. Since $\Gamma=\overline{\left\{c_{1}, c_{2}, \ldots\right\}}$ is compact, there exist $z_{1}, \ldots, z_{N} \in\left\{c_{1}, c_{2}, \ldots\right\}$ so that $\bigcup_{j=1}^{N} D_{\rho}\left(z_{j}, \frac{1}{10 s}\right) \supset \Gamma$. Obviously, $\overline{D_{\rho}\left(z_{j}, \frac{1}{2 s}\right)} \cap K=\emptyset$. For every $j=1, \ldots, N$ we choose $w_{j} \in(\mathbb{Q}+i \mathbb{Q}) \cap\left[D_{\rho}\left(z_{j}, \frac{1}{2 s}\right)-\right.$ $\left.\overline{D_{\rho}\left(z_{j}, \frac{1}{10 s}\right)}\right]$. We consider polygonal lines $E_{j}, j=0,1, \ldots, N$, with vertices in $\mathbb{Q}+i \mathbb{Q}$ such that $E_{j} \subset \mathbb{C} \backslash K$. Every $E_{j}, j=0,, \ldots, N$, starts at $\zeta_{0}$. The curve $E_{0}$ ends at $n+1$ and, for $j=1, \ldots, N$, the curve $E_{j}$ ends at $w_{j}$. There exists $l \in \mathbb{N}$ so that $\operatorname{dist}_{\rho}\left(K, E_{j}\right)>\frac{1}{\ell}$ for all $j=0,1, \ldots, N$.

Then

$$
K \subset L\left(n, \ell, s, N, z_{1}, \ldots, z_{N}, w_{1}, \ldots, w_{N}, E_{0}, E_{1}, \ldots, E_{N}\right),
$$

where $L\left(n, \ell, s, N, z_{1}, \ldots, z_{N}, w_{1}, \ldots, w_{N}, E_{0}, E_{1}, \ldots, E_{N}\right)$ is the set of all $z \in \mathbb{C} \backslash \Omega$ such that $|z| \leq n$, $\operatorname{dist}_{\rho}\left(z, E_{j}\right) \geq \frac{1}{\ell}$ for all $j=0,1, \ldots, N$ and $z \notin D_{\rho}\left(z_{j}, \frac{1}{2 s}\right)$ for all $j=1, \ldots, n$.

The last set is a compact subset of $\mathbb{C}$. The set of all possible such sets is denumerable, because all parameters take values in denumerable sets, $\left\{z_{1}, \ldots, z_{N}\right\}$ are finite subsets of the denumerable set $\left\{c_{1}, c_{2}, \ldots\right\}$, the sets $\left\{w_{1}, \ldots, w_{N}\right\}$ are finite subsets of the denumerable set $\mathbb{Q}+i \mathbb{Q}$ and every $E_{j}(j=0,1, \ldots, N)$ has finitely many vertices taken from $\mathbb{Q}+i \mathbb{Q}$, which is denumerable. Thus, an enumeration of all possible such sets will give the sequence $K_{m}, m=1,2, \ldots$. It remains to verify that $K_{m}^{c}$ is connected. By de Morgan's rule we find

$$
K_{m}^{c}=\Omega \cup\{z \in \mathbb{C}:|z|>n\} \cup\left[\bigcup_{j=0}^{N}\left\{z \in \mathbb{C}: \operatorname{dist}_{\rho}\left(z, E_{j}\right)<\frac{1}{\ell}\right\}\right] \cup\left[\bigcup_{j=1}^{N} D_{\rho}\left(z_{j}, \frac{1}{2 s}\right)\right] .
$$


The sets appearing in this union are all connected. It is important to see how they intersect each other. The point $\zeta_{0}$ is in $\Omega$ and $\zeta_{0} \in\left\{z \in \mathbb{C}: \operatorname{dist}_{\rho}\left(z, E_{j}\right)<\frac{1}{\ell}\right\}$ because $E_{j}$ starts at $\zeta_{0}$. We also have $n+1 \in\{z \in \mathbb{C}:|z|>n\}$ and $n+1 \in$ $\left\{z \in \mathbb{C}: \operatorname{dist}_{\rho}\left(z, E_{0}\right)<\frac{1}{\ell}\right\}$ because $E_{0}$ ends at $n+1$. Therefore,

$$
\Omega \cup\{z \in \mathbb{C}:|z|>n\} \cup\left[\bigcup_{j=0}^{N}\left\{z \in \mathbb{C}: \operatorname{dist}_{\rho}\left(z, E_{j}\right)<\frac{1}{e}\right\}\right]
$$

is connected.

Finally, for $j=1, \ldots, N$ we have $w_{j} \in D_{\rho}\left(z_{j}, \frac{1}{2 s}\right)$ and $w_{j} \in\left\{z \in \mathbb{C}: \operatorname{dist}_{\rho}\left(z, E_{j}\right)\right.$ $\left.<\frac{1}{\ell}\right\}$ because $E_{j}$ ends at $w_{j}$. Thus, $K_{m}^{c}$ is connected. We also notice that $K_{m} \cap \Omega$ $=\emptyset$ and $K_{m} \cap \Gamma=\emptyset$ since

$$
\Gamma \subset \bigcup_{j=1}^{N} D_{\rho}\left(z_{j}, \frac{1}{2 s}\right)
$$

The proof is complete.

Under the above assumptions we have the following two lemmas of double approximation.

Lemma 2.3. Let $K \subset \mathbb{C}$ be compact with $K \cap(\Omega \cup \Gamma)=\emptyset$ and $K^{c}$ connected; let $h: K \rightarrow \mathbb{C}$ be a function continuous on $K$ and holomorphic in $K^{0}$. Let $L \subset \Omega$ be compact and $\varepsilon>0$. Then there exists a rational function $g$ with poles included in $\left\{c_{1}, c_{2}, \ldots\right\}$ such that $g(\infty)=0, \sup _{z \in L}|g(z)|<\varepsilon$ and $\sup _{z \in K}|g(z)-h(z)|<\varepsilon$.

Proof. Without loss of generality we assume that each component of $(\mathbb{C} \cup\{\infty\}) \backslash L$ contains at least one component of $(\mathbb{C} \cup\{\infty\}) \backslash \Omega=F$. Since $\overline{\bigcup_{\ell=1}^{\infty} F_{\ell}}=F$ and every component of $(\mathbb{C} \cup\{\infty\}) \backslash L$ is an open set, it follows that every component of $(\mathbb{C} \cup\{\infty\}) \backslash L$ meets at least one $F_{\ell}, \ell=1,2, \ldots$. Since $c_{\ell} \in F_{\ell}$ and $F_{\ell}$ is connected, it follows that every component of $(\mathbb{C} \cup\{\infty\}) \backslash L$ contains at least one $c_{\ell}, \ell=1,2, \ldots$. The components of $(\mathbb{C} \cup\{\infty\}) \backslash L$ form an open cover of the compact set $K$; therefore, a finite number of them, $V_{1}, \ldots, V_{N}$, suffice to cover $K$. Now $V_{j} \backslash K$ is connected, because $V_{j}$ is connected and the compact set $K$ has connected complement. Therefore, the function

$$
\varphi=\left\{\begin{array}{lll}
h & \text { on } & K \\
0 & \text { on } & \left(V_{1} \cup \cdots \cup V_{N}\right)^{c} \cup\{\infty\}
\end{array}\right.
$$

can be approximated by a rational function $\widetilde{g}$ with poles only at $c_{\ell} \in V_{1} \cup \cdots \cup V_{N}$ so that $\sup _{z \in K \cup\{\infty\} \cup\left(V_{1} \cup \cdots \cup V_{N}\right)^{c}}|\varphi(z)-\widetilde{g}(z)|<\frac{\varepsilon}{2}$.

We set $g=\widetilde{g}-\widetilde{g}(\infty)$, and we are done.

Remark. By Mergelyan's theorem the function $h$ can be approximated on $K$ by a polynomial. Thus, the function to be approximated is holomorphic in an open set containing $K \cup\{\infty\} \cup\left(V_{1} \cup \cdots \cup V_{N}\right)^{c}$. It remains, in the previous proof, to apply an extension of Runge's Theorem valid for open sets containing $\infty$. This is easily proven using a composition with a Mobius transformation.

Finally, under the above assumptions, we have the following lemma of double approximation at the level of functions and their derivatives. 
Lemma 2.4. Let $K \subset \mathbb{C}$ be compact, $K \cap(\Omega \cup \Gamma)=\emptyset$, with connected complement and $h$ a holomorphic function in an open set containing $K$. Let $L \subset \Omega$ be compact, $M \in \mathbb{N}$ and $\varepsilon>0$. Then there exists a rational function $g$ with poles included in $\left\{c_{1}, c_{2}, \ldots\right\}$, so that $g(\infty)=0, \sup _{z \in L}\left|g^{(\ell)}(z)\right|<\varepsilon$ and $\sup _{z \in K}\left|g^{(\ell)}(z)-h^{(\ell)}(z)\right|<\varepsilon$ for all $\ell=0,1, \ldots, M$.

Remark. If $\infty \in L$, then $g^{(\ell)}(\infty)=\lim _{z \rightarrow \infty} g^{(\ell)}(z)$.

Proof. According to the proof of Lemma 2.3 we have the disjoint compact sets $K$ and $\left(V_{1} \cup \cdots \cup V_{N}\right)^{c} \cup\{\infty\}$. Let $\xi=\operatorname{dist}_{\rho}\left(K,\left(V_{1} \cup \cdots \cup V_{N}\right)^{c} \cup\{\infty\}\right)>0$. Every component of the complement of $K \cup\left(V_{1} \cup \cdots \cup V_{N}\right)^{c} \cup\{\infty\}$ contains some of $c_{\ell_{1}}, \ldots, c_{\ell_{T}}$ which belong to $V_{1} \cup \cdots \cup V_{N}$.

Let $S$ be an open set containing $K$ where $h$ is holomorphic. According to Lemma 2.1, there exists an open set $G_{1} \subset \mathbb{C} \cup\{\infty\}$ with $K \subset G_{1} \subset\{z \in S$ : $\left.\operatorname{dist}_{\rho}(z, K)<\min \left(\frac{\xi}{3}, \operatorname{dist}_{\rho}(K, \Gamma)\right)\right\}$ and $G_{1}^{c}$ is connected. (The last claim follows if we apply Lemma 2.1 with $A=\{\infty\}$.)

Also, there exists an open set $G_{2} \subset \mathbb{C} \cup\{\infty\}$ with $\left(V_{1} \cup \cdots \cup V_{N}\right)^{c} \cup\{\infty\} \subset G_{2}$ and $G_{2} \subset\left\{z \in \mathbb{C} \cup\{\infty\}: \operatorname{dist}_{\rho}\left(z,\left(V_{1} \cup \cdots \cup V_{N}\right)^{c} \cup\{\infty\}\right)<\frac{\xi}{3}\right\}$ so that every component of $(\mathbb{C} \cup\{\infty\}) \backslash G_{2}$ meets $\left\{c_{\ell_{1}}, \ldots, c_{\ell_{T}}\right\}$.

Then $G_{1}$ and $G_{2}$ are disjoint and the function

$$
\varphi=\left\{\begin{array}{lll}
h & \text { on } & G_{1} \\
0 & \text { on } & G_{2}
\end{array}\right.
$$

is the limit of a sequence of rational functions $\widetilde{\omega}_{n}, n=1,2, \ldots$, with poles included in $\left\{c_{\ell_{1}}, \ldots, c_{\ell_{T}}\right\}$, where the convergence is uniform on each compact subset of $G_{1} \cup$ $G_{2}$.

We set $\omega_{n}=\widetilde{\omega}_{n}-\widetilde{\omega}_{n}(\infty)$. Then $\omega_{n} \rightarrow \varphi$ compactly in $G_{1} \cup G_{2}$, since $\infty \in G_{2}$. We also have $w_{n}(\infty)=0$. Weierstrass' Theorem applied to the open set $G_{1} \cup G_{2}$ implies that the derivatives $\omega_{n}^{(\ell)}$ converge to $\varphi^{(\ell)}$ compactly in $G_{1} \cup G_{2}$, for every order $\ell$. We set $g=\omega_{n}$, for $n$ large enough, and we have the result.

\section{MAin RESULT}

This section will be an application of the abstract theory of universal series [1] combined with the preliminary results established in the previous section. We present some of the abstract theory we need. This is taken from [1, 1.2 labeled universal series I and 1.3 labeled universal series II, with the simplification $L=$ $\bigcup_{m>1} L_{m}$ to be a singleton and that the scalar field is $\mathbb{C}$.

Denote by $E$ a complete metrizable topological vector space whose topology is induced by a translation-invariant metric $d$. Suppose that for every $n \in \mathbb{N} \cup\{0\}$ there exist an element $e_{n} \in E$ and a function $\Phi_{n}: E \rightarrow \mathbb{C}$ which is linear and continuous.

Let $G=\left\{a=\left(a_{n}\right)_{n=0}^{\infty}, a_{n} \in \mathbb{C}, \exists n_{0}\right.$ so that $\left.a_{n}=0 \forall n \geq n_{0}\right\}$. For every $a \in G$ we write $g_{a}=\sum_{j=0}^{\infty} a_{j} e_{j} \in E$.

In the sequel we shall assume that the set $\left\{g_{a}: a \in G\right\}$ is dense in $E$.

We also assume that for every $a \in G, a=\sum_{j=0}^{\infty} a_{j} e_{j}$, we have $\Phi_{n}(a)=a_{n}$ for all $n=0,1,2, \ldots$. It follows that for every $a \in G$ the set $\left\{n: \Phi_{n}(a) \neq 0\right\}$ is finite.

Also let $\left(X_{k}\right)_{k \geq 1}$ be a sequence of metrizable topological vector spaces over $\mathbb{C}$, equipped with translation-invariant metrics $\rho_{k}$. For any $k \geq 1$, we consider a sequence $\left(x_{k, n}\right)_{n=0}^{\infty}$ of elements of $X_{k}$. 
Definition 3.1. Under the above assumptions an element $f \in E$ belongs to the class $U_{E}$ (resp., to the class $U_{E}^{\mu}$, where $\mu$ is an infinite subset of $\mathbb{N} \cup\{0\}$ ) if, for every $k \geq 1$ and every $x \in X_{k}$, there exists a sequence $\left(\lambda_{n}\right)_{n=1}^{\infty}, \lambda_{n} \in \mathbb{N}\left(\right.$ resp. $\left.\lambda_{n} \in \mu\right)$, for all $n=1,2, \ldots$, such that

i) $\rho_{k}\left(\sum_{j=0}^{\lambda_{n}} \Phi_{j}(f) x_{k, j}, x\right) \rightarrow 0$, as $n \rightarrow+\infty$, and

ii) $d\left(\sum_{j=0}^{\lambda_{n}} \Phi_{j}(f) e_{j}, f\right) \rightarrow 0$, as $n \rightarrow+\infty$.

Then we have the following:

Theorem $3.2([1])$. Under the above assumptions, the following are equivalent:

1) $U_{E} \neq \emptyset$.

2) For every $k \geq 1$, every $x \in X_{k}$ and every $\varepsilon>0$, there exist $n \in \mathbb{N}$ and $a_{0}, \ldots, a_{n} \in \mathbb{C}$ so that $\rho_{k}\left(\sum_{j=0}^{n} a_{j} x_{k, j}, x\right)<\varepsilon$ and $d\left(\sum_{j=0}^{n} a_{j} e_{j}, 0\right)<\varepsilon$.

3) For every infinite set $\mu \subset \mathbb{N} \cup\{0\}$, the class $U_{E}^{\mu}$ is a dense $G_{\delta}$ subset of $E$.

4) For every infinite set $\mu \subset \mathbb{N} \cup\{0\}$, the class $U_{E}^{\mu}$ contains, except 0 , a dense subspace of $E$.

Let $F$ be a closed subset of $\mathbb{C} \cup\{\infty\}$ such that $\Omega=(\mathbb{C} \cup\{\infty\}) \backslash F$ is connected. We assume that among the components of $F$ we may choose denumerably many $F_{1}, F_{2}, \ldots$ which are compact subsets of $\mathbb{C}$ such that Condition $(A)$ is satisfied. We also fix a point $c_{\ell}$ in $F_{\ell}, \ell=1,2, \ldots$, and we set $\Gamma=\overline{\left\{c_{\ell}, \ell \in \mathbb{N}\right\}}$, where the closure is taken in $\mathbb{C} \cup\{\infty\}$. We emphasize that $c_{\ell} \neq \infty$ for every $\ell \in \mathbb{N}$. We consider the vector space of all rational functions $q$ with poles included in $\left\{c_{\ell}, \ell=1,2, \ldots\right\}$ and such that $q(\infty)=0$. We endow this space with the topology of uniform convergence on compact subsets of $\Omega$ and let $(E, d)$ denote its completion. If $\infty \notin F$, in which case $\infty \in \Omega$, then it is easy to see that $E=H_{0}(\Omega)=\{f \in H(\Omega): f(\infty)=0\}$ endowed with the topology of uniform convergence on compact sets. This follows by Runge's Theorem. Indeed, if $L \subset \Omega$ is a compact subset we may assume that every component of $(\mathbb{C} \cup\{\infty\}) \backslash L$ contains a component of $F=(\mathbb{C} \cup\{\infty\}) \backslash \Omega$. Since $\bigcup_{\ell=1}^{\infty} F_{\ell}$ is dense in $F$ and any component of $(\mathbb{C} \cup\{\infty\}) \backslash L$ is open, it follows that it meets some $F_{\ell}, \ell \in \mathbb{N}$. Since this $F_{\ell}$ is a connected subset of $(\mathbb{C} \cup\{\infty\}) \backslash L$, it follows that it is contained in the given component of $(\mathbb{C} \cup\{\infty\}) \backslash L$. Thus, every component of $(\mathbb{C} \cup\{\infty\}) \backslash L$ contains some $c_{\ell}, \ell \in \mathbb{N}$. Thus, Runge's Theorem easily yields that $E=H_{0}(\Omega)$ in the case $\infty \notin F$. If $\infty \in F$, in which case $\infty \notin \Omega$, then $E=H(\Omega)$; this follows easily by Runge's Theorem and the fact that a subsequence of points $\tilde{c}_{\ell} \in F_{\ell}, \ell \in \mathbb{N}$, converges to $\infty$. Thus, if $L \subset \Omega$ is a compact set, the component of $(\mathbb{C} \cup\{\infty\}) \backslash L$ containing $\infty$, being an open set, necessarily contains some $\tilde{c}_{\ell}, \ell \in \mathbb{N}$. Since $F_{\ell}$ is a connected subset of $(\mathbb{C} \cup\{\infty\}) \backslash L$ and $c_{\ell}, \tilde{c}_{\ell} \in F_{\ell}$, it follows that the component of $(\mathbb{C} \cup\{\infty\}) \backslash L$ containing $\infty$ necessarily contains some $c_{\ell}, \ell \in \mathbb{N}$.

Next we consider the family of functions $\frac{1}{\left(z-c_{\ell}\right)^{m}} ; \ell=1,2, \ldots, m=1,2, \ldots$ This is a denumerable subset of $E$, and therefore it can take the form $\left\{e_{n}, n=0,1,2, \ldots\right\}$. The ordering may be arbitrary, but will be fixed.

We notice that $\left\{g_{a}: a \in G\right\}$ coincides with the set of rational functions $q$ with poles in $\left\{c_{\ell} ; \ell \in \mathbb{N}\right\}$ and satisfying $q(\infty)=0$. This is dense in its completion $E$.

For $f \in E$ and $\ell \in \mathbb{N}$ we consider a closed polygonal curve $\gamma_{\ell}$ in $\Omega \cap \mathbb{C}$ such that $\operatorname{Ind}\left(\gamma_{\ell}, c_{\ell}\right)=-1$ and $\operatorname{Ind}\left(\gamma_{\ell}, c_{j}\right)=0$ for $j \neq \ell$. This can be done because of our assumption that $F_{\ell}$ is far from $F \backslash F_{\ell}$. We consider the function $f_{\ell}(z)=\frac{1}{2 \pi i} \int_{\gamma_{\ell}} \frac{f(\zeta)}{\zeta-z} d \zeta$, which extends holomorphically in $(\mathbb{C} \cup\{\infty\}) \backslash F_{\ell}$ and satisfies $f_{\ell}(\infty)=0$. It is easy 
to see that $f_{\ell}$ is independent of $\gamma_{\ell}$ provided that $\operatorname{Ind}\left(\gamma_{\ell}, c_{\ell}\right)=-1$ and $\operatorname{Ind}\left(\gamma_{\ell}, c_{j}\right)=0$ for $j \neq \ell$. Because $f_{\ell}(z)$ is given as an integral of $f$ on a compact subset of $\Omega \cap \mathbb{C}$, one can easily see that the map $E \ni f \rightarrow f_{\ell} \in H_{0}\left((\mathbb{C} \cup\{\infty\}) \backslash F_{\ell}\right)$ is continuous when $H_{0}\left((\mathbb{C} \cup\{\infty\}) \backslash F_{\ell}\right)$ is endowed with the topology of uniform convergence on compact subsets of $(\mathbb{C} \cup\{\infty\}) \backslash F_{\ell}$.

Furthermore, since $F_{\ell}$ is compact, it is contained in a closed disc $\bar{D}_{\ell}$ centered in $c_{\ell}$. Then in $(\mathbb{C} \cup\{\infty\}) \backslash \bar{D}_{\ell}$ we have a Laurent expansion

$$
f_{\ell}(z)=\sum_{m=1}^{\infty} a_{m}\left(f_{\ell}\right) \frac{1}{\left(z-c_{\ell}\right)^{m}} .
$$

It is well-known that the maps $H_{0}\left((\mathbb{C} \cup\{\infty\}) \backslash \bar{D}_{\ell}\right) \ni f_{\ell} \rightarrow a_{m}\left(f_{\ell}\right) \in \mathbb{C}$ are continuous. By composition the maps $E \ni f \rightarrow a_{m}\left(f_{\ell}\right) \in \mathbb{C}$ are continuous.

Now for every $\ell \in \mathbb{N}$ and $m=1,2, \ldots$ there exists a unique $n \in\{0,1,2, \ldots\}$ so that $\frac{1}{\left(z-c_{\ell}\right)^{m}}=e_{n}$ and conversely. We set $\Phi_{n}(f)=a_{m}\left(f_{\ell}\right)$. Obviously the maps $\Phi_{n}: E \rightarrow \mathbb{C}$ are linear and continuous.

In order to be able to apply the abstract theory it remains to determine the spaces $X_{k}, k=1,2, \ldots$, and the vectors $x_{k, n} \in X_{k}$. We will make two choices for the spaces $X_{k}, k=1,2, \ldots$ According to Lemma 2.2 there exists a sequence $K_{m} \subset \mathbb{C}$, $m=1,2, \ldots$, of compact sets, $K_{m} \cap(\Omega \cup \Gamma)=\emptyset$ with connected complement so that every compact set $K \subset \mathbb{C}, K \cap(\Omega \cup \Gamma)=\emptyset$, with connected complement is contained in some $K_{m}$. One choice of $X_{k}$ will be $X_{k}=A\left(K_{k}\right)=\left\{h: K_{k} \rightarrow \mathbb{C}\right.$ continuous on $K_{k}$ and holomorphic in $\left.K_{k}^{0}\right\}$ endowed with the supremum norm.

Another choice of $X_{k}$, denoted by $X_{k}^{\prime}$, will be the set of holomorphic functions on some (varying) neighborhood of $K_{k}$ endowed with the standard metric $\rho_{k}$ com-

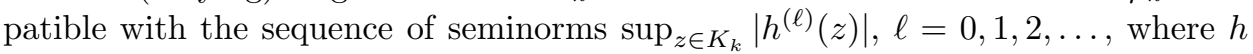
denotes any holomorphic function on some neighborhood of $K_{k}$.

The vector $x_{k, n}, n=0,1, \ldots$, will always be $x_{k, n}=\frac{1}{\left(z-c_{\ell}\right)^{m}}$ seen as an element of $X_{k}$, or $X_{k}^{\prime}$, where $n, \ell, m$ are related so that $\frac{1}{\left(z-c_{\ell}\right)^{m}} \in E$ equals $e_{n}$. Then we denote $\ell=\ell_{n}$ and $m=m_{n}$.

In order to establish that $U_{E}^{\mu}$ is non-empty it suffices to verify condition 2 of Theorem 3.2. In the case of the spaces $X_{k}$, this has been done in Lemma 2.3. In the case of the spaces $X_{k}^{\prime}$ this has been done in Lemma 2.4. Thus, in either case, $U_{E}^{\mu}$ is non-void, in fact dense, is a $G_{\delta}$ set and contains a dense vector subspace of $E$ except 0 .

Finally, the approximations on each compact set $K_{m}, m=1,2, \ldots$, imply approximation on all compact sets $K \subset \mathbb{C}, K \cap(\Omega \cup \Gamma)=\emptyset$, with connected complement.

If $h \in A(K)$, by Mergelyan's theorem, $h$ may be uniformly approximated on $K$ by a polynomial $p$. Now $K \subset K_{m}$, for some $m$. But $p$ is defined on $K_{m}$ and can be approximated on it by partial sums of our universal series. So by the triangle inequality the same holds for $h$ on $K$. This gives the result in the case of the spaces $X_{k}$.

In the case of $X_{k}^{\prime}$ we argue as follows:

Let $K \subset \mathbb{C}$ be compact and $K \cap(\Omega \cup \Gamma)=\emptyset$ with connected complement; let $V \subset \mathbb{C}$ be an open set, $K \subset V$ and let $h: V \rightarrow \mathbb{C}$ be holomorphic in $V$. According to Lemma 2.1 (see also [5], 2]) we may assume that $(\mathbb{C} \cup\{\infty\}) \backslash V$ is connected. By Runge's Theorem, there exists a sequence of polynomials $p_{n}, n=1,2, \ldots$, converging to $h$ uniformly on compact subsets of $V$. By Weierstrass' Theorem we 
have $p_{n}^{(\ell)} \rightarrow h^{(\ell)}$, as $n \rightarrow+\infty$, uniformly on compact subsets of $V$, for derivatives of all orders $\ell=0,1, \ldots$.

Thus we find $n$ large enough so that $p=p_{n}$ satisfies $\sup _{z \in K}\left|h^{(\ell)}(z)-p^{(\ell)}(z)\right|<\frac{\varepsilon}{2}$ for $\ell=0,1, \ldots, N$.

Now $K \subset K_{m}$ for some $m$ and $p$, being a polynomial, is defined on $K_{m}$; but on $K_{m}$ we can approximate $p^{(\ell)}, \ell=0, \ldots, N$, by the derivatives of a partial sum of our universal series. Thus, we have proven the following two theorems.

Theorem 3.3. Let $\Omega$ be a domain of infinite connectivity and such that Condition $(A)$ is satisfied. If $\infty \in \Omega$, then $E=H_{0}(\Omega)$. If $\infty \notin \Omega$, then $E=H(\Omega)$. Let $\mu \subset \mathbb{N} \cup\{0\}$ be an infinite set. There exists $f \in E$ so that the sequence $T_{n}(f)$ defined by $T_{n}(f)(z)=\sum_{j=0}^{n} \Phi_{j}(f) \frac{1}{\left(z-c_{i_{j}}\right)^{m}}$ for $n=0,1, \ldots$ has the following property:

For every compact set $K \subset \mathbb{C}, K \cap(\Omega \cup \Gamma)=\emptyset$, with connected complement and every $h: K \rightarrow \mathbb{C}$ continuous on $K$ and holomorphic in $K^{0}$, there exists a sequence $\lambda_{n} \in \mu, n=1,2, \ldots$, so that

$$
\begin{aligned}
T_{\lambda_{n}}(f) \rightarrow & f \text { uniformly on compact subsets of } \Omega, \text { as } n \rightarrow+\infty \text { and } \\
& T_{\lambda_{n}}(f) \rightarrow h \text { uniformly on } K, \text { as } n \rightarrow+\infty .
\end{aligned}
$$

The set of such functions $f$ is $a G_{\delta}$ and dense subset of $E$ and contains a dense vector subspace of $E$ except 0 .

Theorem 3.4. Let $\Omega$ be a domain of infinite connectivity and such that Condition (A) is satisfied. If $\infty \in \Omega$, then $E=H_{0}(\Omega)$. If $\infty \notin \Omega$, then $E=H(\Omega)$. Let $\mu \subset \mathbb{N} \cup\{0\}$ be an infinite set. There exists $f \in E$ so that the sequence $T_{n}(f)(z)=\sum_{j=0}^{n} \Phi_{j}(f) \frac{1}{\left(z-c_{\ell_{j}}\right)^{m_{j}}}$ for $n=0,1,2, \ldots$ has the following property:

For every compact set $K \subset \mathbb{C}, K \cap(\Omega \cup \Gamma)=\emptyset$, with connected complement and every complex function $h$ holomorphic in some open set containing $K$, there exists a sequence $\lambda_{n} \in \mu, n=1,2, \ldots$, so that

$$
T_{\lambda_{n}}(f) \rightarrow f \text { uniformly on compact subsets of } \Omega \text {, as } n \rightarrow+\infty
$$

and $\left[T_{\lambda_{n}}(f)\right]^{(\ell)} \rightarrow h^{(\ell)}$ uniformly on $K$, as $n \rightarrow+\infty$, for derivatives of all orders $\ell=0,1,2, \ldots$.

The set of such functions $f$ is $a G_{\delta}$ and dense subset of $E$ and contains a dense vector subspace of $E$ except 0 .

\section{ACKNOWLEDGEMENTS}

The author wishes to thank the anonymous referee and A. Katavolos for improving the presentation of this article.

\section{References}

[1] F. Bayart, K.-G. Grosse-Erdmann, V. Nestoridis, and C. Papadimitropoulos, Abstract theory of universal series and applications, Proc. Lond. Math. Soc. (3) 96 (2008), no. 2, 417-463, DOI 10.1112/plms/pdm043. MR2396846(2009j:30006)

[2] G. Costakis, Some remarks on universal functions and Taylor series, Math. Proc. Cambridge Philos. Soc. 128 (2000), no. 1, 157-175, DOI 10.1017/S0305004199003886. MR1724436 (2001g:30002)

[3] G. Costakis, V. Nestoridis, and I. Papadoperakis, Universal Laurent series, Proc. Edinb. Math. Soc. (2) 48 (2005), no. 3, 571-583, DOI 10.1017/S0013091504000495. MR2171185 (2006g:30005)

[4] Wolfgang Gehlen, Wolfgang Luh, and Jürgen Müller, On the existence of O-universal functions, Complex Variables Theory Appl. 41 (2000), no. 1, 81-90. MR1758599 (2001a:30003) 
[5] Karl-Goswin Große-Erdmann, Holomorphe Monster und universelle Funktionen (German), Mitt. Math. Sem. Giessen 176 (1987), iv+84. Dissertation, University of Trier, Trier, 1987. MR.877464 (88i:30060)

[6] Karl-Goswin Grosse-Erdmann, Universal families and hypercyclic operators, Bull. Amer. Math. Soc. (N.S.) 36 (1999), no. 3, 345-381, DOI 10.1090/S0273-0979-99-00788-0. MR,1685272(2000c:47001)

[7] Jean-Pierre Kahane, Baire's category theorem and trigonometric series, J. Anal. Math. 80 (2000), 143-182, DOI 10.1007/BF02791536. MR1771526(2001f:42012)

[8] Daniel Mayenberger and Vagia Vlachou, Construction of a universal Laurent series, Comput. Methods Funct. Theory 5 (2005), no. 2, 365-372. MR2205419 (2007c:30002)

[9] A. Melas and V. Nestoridis, Universality of Taylor series as a generic property of holomorphic functions, Adv. Math. 157 (2001), no. 2, 138-176, DOI 10.1006/aima.2000.1955. MR.1813429 (2002e:30002)

[10] Antonios D. Melas, Universal functions on nonsimply connected domains (English, with English and French summaries), Ann. Inst. Fourier (Grenoble) 51 (2001), no. 6, 1539-1551. MR.1870639(2002j:30004)

[11] J. Müller, V. Vlachou, and A. Yavrian, Universal overconvergence and Ostrowski-gaps, Bull. London Math. Soc. 38 (2006), no. 4, 597-606, DOI 10.1112/S0024609306018492. MR2250752 (2007h:30002)

[12] J. Müller, V. Vlachou, and A. Yavrian, Overconvergent series of rational functions and universal Laurent series, J. Anal. Math. 104 (2008), 235-245, DOI 10.1007/s11854-008-0023-7. MR2403436 (2009e:30007)

[13] Vassili Nestoridis, Universal Taylor series (English, with English and French summaries), Ann. Inst. Fourier (Grenoble) 46 (1996), no. 5, 1293-1306. MR1427126 (97k:30001)

[14] Vassili Nestoridis, An extension of the notion of universal Taylor series, Computational methods and function theory 1997 (Nicosia), Ser. Approx. Decompos., vol. 11, World Sci. Publ., River Edge, NJ, 1999, pp. 421-430. MR.1700365 (2000e:30003)

[15] N. Tsirivas, Universal Faber and Taylor series on an unbounded domain of infinite connectivity, Complex Var. Elliptic Equ. 56 (2011), 533-542, DOI 10.1080/17476933.2010.504832. MR2821383(2012h:30192)

[16] V. Vlachou, A universal Taylor series in the doubly connected domain $\mathbb{C} \backslash\{1\}$, Complex Var. Theory Appl. 47 (2002), no. 2, 123-129, DOI 10.1080/02781070290010878. MR1892513 (2002m:30002)

Department of Mathematics, University of Athens, 15784 Panepistemiopolis, Athens, Greece

E-mail address: vnestor@math.uoa.gr 